\title{
Singlet-Doublet Fermionic Dark Matter, Neutrino Mass and Collider Signatures
}

\author{
Subhaditya Bhattacharya* \\ Department of Physics, Indian Institute of Technology Guwahati, North Guwahati, Assam- 781039, India

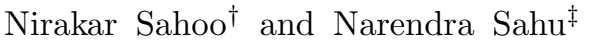 \\ Department of Physics, Indian Institute of Technology, \\ Hyderabad, Kandi, Sangareddy, 502285, Telangana, India
}

\begin{abstract}
We propose a minimal extension of the standard model (SM) by including a scalar triplet with hypercharge 2 and two vector-like leptons: one doublet and a singlet, to explain simulatenously the non-zero neutrino mass and dark matter (DM) content of the Universe. The DM emerges out as a mixture of the neutral component of vector-like lepton doublet and singlet, being odd under a discrete $Z_{2}$ symmetry. After electroweak symmetry breaking the triplet scalar gets an induced vev, which give Majorana masses not only to the light neutrinos but also to the DM. Due to the Majorana mass of DM, the $Z$ mediated elastic scattering with nucleon is forbidden. However, the Higgs mediated direct detection cross-section of the DM gives an excellent opportunity to probe it at Xenon-1T. The DM can not be detected at collider. However, the charged partner of the DM (often next-to-lightest stable particle) can give large dispalced vertex signature at the Large Hadron Collider (LHC).

PACS numbers: $98.80 . \mathrm{Cq}, 12.60 . \mathrm{Fr}$
\end{abstract}

*Electronic address: subhab@iitg.ernet.in

$\dagger$ Electronic address: nirakar.pintu.sahoo@gmail.com

${ }^{\ddagger}$ Electronic address: nsahu@iith.ac.in 


\section{INTRODUCTION}

Astrophysical evidences like galaxy rotation curves, gravitational lensing, large scale structure of the universe and anisotropies in Cosmic Microwave Background Radiation (CMBR) hint towards the existence of an unknown form of non-luminous matter, called dark matter (DM) in our Universe 1, 2. However they imply only about the gravitational property of DM, whose relic abundance is precisely measured by the satellite borne experiments such as WMAP [3] and PLANCK 4 to be $\Omega_{\mathrm{DM}} h^{2}=0.1199 \pm 0.0027$. However, the microscopic nature of DM is still a mystery. In fact, none of the particles in the standard model (SM) mimics the properties of DM. This leads to a rich possibility of DM model building in the beyond SM physics, though the WIMP (Weakly Interacting Massive Particle) paradigm is the most popular one.

Another significant hint of physics beyond the SM came through the discovery of non-zero masses of left-handed neutrinos in the last decade. Long baseline neutrino oscillation experiments [5, 6] confirmed that neutrinos have sub$\mathrm{eV}$ masses and they mix among themselves. This also has compelled particle physicists to explore structures beyond the SM. There are two major issues involving neutrinos: (i) their nature, Dirac or Majorana, (ii) mass hierarchy, normal or inverted. A popular solution for non-zero Majorana masses of active neutrinos is to introduce the seesaw mechanisms [7 9] which are different realisations of the dimension five operator [10]: $L L H H / \Lambda$, where $L$ and $H$ are the lepton and Higgs doublet of the SM and $\Lambda$ is the scale of new physics. After electroweak (EW) symmetry breaking the neutrino mass is given by $M_{\nu}=\langle H\rangle^{2} / \Lambda$. Thus for tiny neutrino mass $M_{\nu} \sim 0.1 \mathrm{eV}$, the new physics scale requires to be pretty heavy $\Lambda \sim 10^{14} \mathrm{GeV}$ when the involved couplings are order 1 . However, $\Lambda$ can be reduced down to a $\mathrm{TeV}$ scales if the couplings are assumed to be smaller.

In an attempt to bring dark matter and neutrino mass mechanisms under one umbrella (for some earlier attempts, see [11 13] ), we consider a minimal type-II seesaw extension of the SM by adding a TeV scale triplet scalar $\Delta$ with hypercharge 2 and consider two vector-like leptons: one $\operatorname{doublet} N \equiv\left(N^{0}, N^{-}\right)^{T}$ and a singlet $\chi$. A $Z_{2}$ symmetry is also imposed under which $N$ and $\chi$ are odd while all other fields are even. As a result the DM emerges out to be a mixed state of singlet and neutral component of the doublet vector-like leptons. Such DM frameworks have been discussed earlier; see for example, refs. [14 25. However, the presence of the triplet adds to some interesting DM phenomenology as we will discuss in this paper. Since the scalar triplet can be light, it contributes to the relic abundance of DM through s-channel resonance on top of $Z$ and $H$ mediation. Moreover, it relaxes the strong constraints coming from direct detection.

The triplet scalar not only couples to the SM lepton and Higgs doublets, but also to the additional vector-like lepton doublet $N$. The Majorana couplings of $\Delta$ with $N, L$ and $H$ is then be given by $f_{N} \Delta N N+f_{L} \Delta L L+\mu \Delta^{\dagger} H H$. Note that if the triplet is heavier than the DM and leptons, it can be integrated out and hence effectively generating the dimension five operators:

$$
\left(\frac{L L H H}{\Lambda}+\frac{N N H H}{\Lambda}\right) \text {, }
$$

where $\Lambda \sim M_{\Delta}$. After EW symmetry breaking $\Delta$ acquires an induced vacuum expectation value (vev) of $\mathcal{O}(1) \mathrm{GeV}$ which in turn give Majorana masses to light neutrinos as well as to $N^{0}$. Since $N^{0}$ is a vector-like Dirac fermion, it can have a Dirac mass too. As a result $N^{0}$ splits up into two pseudo-Dirac fermions, with a mass splitting of sub-GeV order, whose elastic scattering with the nucleon mediated by $Z$-boson is forbidden. This feature of the model leads to a survival of larger region of parameter space from direct search constraints given by the latest data from Xenon-100 [26] and LUX [27. On the other hand, the Higgs mediated elastic scattering of the DM with the nucleon gives an excellent opportunity to detect it at future direct search experiments such as XENON1T [28. It is harder to see the signature of only DM production at collider as they need to recoil against an ISR jet for missing energy. However, the charged partner of the DM (which is next-to-lightest stable particle) can be produced copiously which eventually decays to DM giving rise to leptons and missing energy. More interestingly, the charged companion can also give large displaced vertex signature as we will elaborate.

The paper is arranged as follows: in section II], we discuss model and formalism, mixing in fermionic and in scalar sector; in section III] we explain non-zero neutrino mass in a type II see-saw scenario; relic abundance of DM is illustrated in section IV] inelastic scattering of DM with the nucleus for direct search is presented in section V: section VI is devoted for direct detection through elastic scattering and limits on model parameter space; displaced vertex signature of the charged partner of DM is discussed in sec VII. With a summary of the analysis, we finally conclude in section VIII. 


\section{THE MODEL}

As already been stated in the introduction, we extend the standard model (SM) by introducing two vector like fermions $N^{T}=\left(N^{0}, N^{-}\right)(1,2,-1)$ and $\chi^{0}(1,1,0)$ and a scalar triplet $\Delta(1,3,2)$. The numbers inside the parenthesis are quantum numbers under the SM gauge group $S U(3)_{c} \times S U(2)_{L} \times U(1)_{Y}$. A $Z_{2}$ symmetry is imposed under which $\chi^{0}$ and $N$ are odd, while other fields are even. The relevant Lagrangian involving the additional fields is given by:

$$
\mathcal{L}_{\text {new }}=\bar{N} \not \partial N+\overline{\chi^{0}} \not \partial \chi^{0}+\left(D^{\mu} \Delta\right)^{\dagger}\left(D_{\mu} \Delta\right)+M_{N} \bar{N} N+M_{\chi} \overline{\chi^{0}} \chi^{0}+\mathcal{L}_{y u k}-V(\Delta, H),
$$

where $D_{\mu}$ is the covariant derivative and is given by :

$$
D_{\mu}=\partial_{\mu}-i \frac{g}{2} \tau . W_{\mu}-i g^{\prime} \frac{Y}{2} B_{\mu}
$$

The scalar potential in Eq. (1) is given by

$$
\begin{aligned}
V(\Delta, H) & =-M_{H}^{2} H^{\dagger} H+\lambda_{H}\left(H^{\dagger} H\right)^{2}+\mu_{\Delta}^{2}\left(\Delta^{\dagger} \Delta\right)+\lambda_{\Delta}\left(\Delta^{\dagger} \Delta\right)^{2} \\
& +\lambda_{H \Delta}\left(H^{\dagger} H\right)\left(\Delta^{\dagger} \Delta\right)+\frac{1}{\sqrt{2}}\left[\mu \Delta^{\dagger} H H+\text { h.c. }\right]
\end{aligned}
$$

where $\Delta$ in matrix form is

$$
\Delta=\left(\begin{array}{cc}
\frac{\Delta^{+}}{\sqrt{2}} & \Delta^{++} \\
\Delta^{0} & -\frac{\Delta^{+}}{\sqrt{2}}
\end{array}\right)
$$

We assume that $\mu_{\Delta}^{2}$ is positive. So it doesn't acquire a vacuum expectation value (vev). But it gets an induced vev after EW phase transition. The vev of $\Delta$ is given by

$$
\langle\Delta\rangle \equiv u_{\Delta} \approx-\frac{\mu v^{2}}{2\left(\mu_{\Delta}^{2}+\lambda_{H \Delta} v^{2} / 2\right)}
$$

where $v$ is the vev of Higgs field and its value is $174 \mathrm{GeV}$.

The Yukawa interaction in Eq. (1) is given by:

$$
\mathcal{L}_{y u k}=\frac{1}{\sqrt{2}}\left[\left(f_{L}\right)_{\alpha \beta} \overline{L_{\alpha}^{c}} i \tau_{2} \Delta L_{\beta}+f_{N} \overline{N^{c}} i \tau_{2} \Delta N+\text { h.c }\right]+\left[Y \bar{N} \widetilde{H} \chi^{0}+\text { h.c. }\right]
$$

which importantly inherit the source of neutrino masses and DM-SM interactions.

\section{A. Singlet-doublet fermion mixing}

After electroweak phase transition vev of Higgs field introduces a mixing between $N^{0}$ and $\chi^{0}$. The mass matrix is given by

$$
\mathcal{M}=\left(\begin{array}{ll}
M_{\chi} & m_{D} \\
m_{D} & M_{N}
\end{array}\right)
$$

where $m_{D}=Y v$. Diagonalizing the above mass matrix we get two mass eigenvalues:

$$
\begin{gathered}
M_{1} \approx M_{\chi}-\frac{m_{D}^{2}}{M_{N}-M_{\chi}} \\
M_{2} \approx M_{N}+\frac{m_{D}^{2}}{M_{N}-M_{\chi}}
\end{gathered}
$$

where we have assumed $m_{D}<<M_{N}, M_{\chi}$. The corresponding mass eigenstates are given by:

$$
\begin{gathered}
N_{1}=\cos \theta \chi^{0}+\sin \theta N^{0} \\
N_{2}=\cos \theta N^{0}-\sin \theta \chi^{0},
\end{gathered}
$$


where the mixing angle is given by:

$$
\tan 2 \theta=\frac{2 m_{D}}{M_{N}-M_{\chi}} .
$$

Due to the imposed $Z_{2}$ symmetry, the lightest odd particle remains stable. We choose this to be $N_{1}$ and hence becomes a viable candidate of DM. The next-to-lightest particle is the $Z_{2}$ odd charged lepton $N^{-}$whose mass in terms of $M_{1}, M_{2}$ and $\theta$ is given by:

$$
M^{ \pm}=M_{1} \sin ^{2} \theta+M_{2} \cos ^{2} \theta \simeq M_{N} .
$$

From Eq. 9, we see that $Y$ and $\sin \theta$ are not two independent parameters. They are related by:

$$
Y=\frac{\Delta M \sin 2 \theta}{2 v},
$$

with $\Delta M=M_{2}-M_{1}$. We use $\sin \theta$ as an independent parameter in our analysis. We will see that the mixing angle plays a vital role in the DM phenomenology. In particular, the relic abundance of DM gives an upper bound on the mixing angle to be $\sin \theta \lesssim 0.2$. For larger mixing angle the relic abundance is less than the observed value in all parameter space. We also found that a lower bound on $\sin \theta$ comes from the decay of $N_{2}$ and $N^{-}$after they freeze out from the thermal bath. In principle these particles can decay on, before or after the DM $\left(N_{1}\right)$ freezes out depending on the mixing angle. In the worst case, $N_{2}$ and $N^{-}$have to decay before the onset of Big-Bang nucleosynthesis. In that case, the lower bound on the mixing angle is very much relaxed and the out-of-equilibrium decay of $N_{2}$ and $N^{-}$ will produce an additional abundance of DM. Therefore, in what follows, we demand that $N_{2}$ and $N^{-}$decay on or before the freeze out of DM $\left(N_{1}\right)$. As a result we get a stronger lower bound on $\sin \theta$, which of course depends on their masses.

If the mass splitting between $N^{-}$and $N_{1}$ is larger than $W^{ \pm}$-boson mass, then $N^{-}$decay preferably through the two body process: $N^{-} \rightarrow N_{1}+W^{-}$. However, if the mass splitting between $N^{-}$and $N_{1}$ is less than $W^{ \pm}$-boson mass then $N^{-}$decay through the three body process: $N^{-} \rightarrow N_{1} \ell^{-} \overline{\nu_{\ell}}$. For the latter case, we get a stronger lower bound on the mixing angle than the former. The decay width of $N^{-}$is given by [14:

$$
\Gamma=\frac{G_{F}^{2} \sin ^{2} \theta}{24 \pi^{3}} M_{N}^{5} I
$$

where $G_{F}$ is the Fermi coupling constant and $I$ is given as:

$$
I=\frac{1}{4} \lambda^{1 / 2}\left(1, a^{2}, b^{2}\right) F_{1}(a, b)+6 F_{2}(a, b) \ln \left(\frac{2 a}{1+a^{2}-b^{2}-\lambda^{1 / 2}\left(1, a^{2}, b^{2}\right)}\right) .
$$

In the above Equation $F_{1}(a, b)$ and $F_{2}(a, b)$ are two polynomials of $a=M_{1} / M_{N}$ and $b=m_{\ell} / M_{N}$, where $m_{\ell}$ is the charged lepton mass. Up to $\mathcal{O}\left(b^{2}\right)$, these two polynomials are given by

$$
\begin{aligned}
& F_{1}(a, b)=\left(a^{6}-2 a^{5}-7 a^{4}\left(1+b^{2}\right)+10 a^{3}\left(b^{2}-2\right)+a^{2}\left(12 b^{2}-7\right)+\left(3 b^{2}-1\right)\right) \\
& F_{2}(a, b)=\left(a^{5}+a^{4}+a^{3}\left(1-2 b^{2}\right)\right) .
\end{aligned}
$$

In Eq. 13, $\lambda^{1 / 2}=\sqrt{1+a^{4}+b^{4}-2 a^{2}-2 b^{2}-2 a^{2} b^{2}}$ defines the phase space. In the limit $b=m_{\ell} / M_{N} \rightarrow 1-a=$ $\Delta M / M_{N}, \lambda^{1 / 2}$ goes to zero and hence $I \rightarrow 0$. The life time of $N^{-}$is then given by $\tau \equiv \Gamma^{-1}$. We take the freeze out temperature of DM to be $T_{f}=M_{1} / 20$. Since the DM freezes out during radiation dominated era, the corresponding time of DM freeze-out is given by :

$$
t_{f}=0.301 g_{\star}^{-1 / 2} \frac{m_{\mathrm{pl}}}{T_{f}^{2}},
$$

where $g_{\star}$ is the effective massless degrees of freedom at a temperature $T_{f}$ and $m_{\mathrm{pl}}$ is the Planck mass. Demanding that $N^{-}$should decay before the DM freezes out (i.e. $\tau \lesssim t_{f}$ ) we get

$$
\sin \theta \gtrsim 1.1789 \times 10^{-5}\left(\frac{1.375 \times 10^{-5}}{I}\right)^{1 / 2}\left(\frac{200 \mathrm{GeV}}{M_{N}}\right)^{5 / 2}\left(\frac{g_{\star}}{106.75}\right)^{1 / 4}\left(\frac{M_{1}}{180 \mathrm{GeV}}\right) .
$$

Notice that the lower bound on the mixing angle depends on the mass of $N^{-}$and $N_{1}$. For a typical value of $M_{N}=200$ $\mathrm{GeV}, M_{1}=180 \mathrm{GeV}$, we get $\sin \theta \gtrsim 1.17 \times 10^{-5}$. Since $\tau$ is inversely proportional to $M_{N}^{5}$, larger the mass, smaller will be the lower bound on the mixing angle. We will come back to this issue while calculating the relic abundance of DM in section IV. 


\section{B. Doublet-triplet scalar mixing}

In the scalar sector, the model constitutes a doublet and a triplet. The quantum fluctuations around the vacuum is given as:

$$
\phi^{0}=\frac{1}{\sqrt{2}}\left(v+h^{0}+i \xi^{0}\right), \Delta^{0}=\frac{1}{\sqrt{2}}\left(u_{\Delta}+\delta^{0}+i \eta^{0}\right)
$$

The mass matrix is given as :

$$
\mathcal{M}_{s c}^{2}=\left(\begin{array}{cc}
M_{H}^{2} & \mu v / 2 \\
\mu v / 2 & M_{\Delta}^{2}
\end{array}\right)
$$

where $M_{\Delta}^{2}=\mu_{\Delta}^{2}+\lambda_{H \Delta} v^{2} / 2$. The two neutral Higgs fields (CP - even) mass eigenstates are given by

$$
H_{1}=\cos \theta_{0} h^{0}+\sin \theta_{0} \delta^{0}, H_{2}=-\sin \theta_{0} h^{0}+\cos \theta_{0} \delta^{0}
$$

where $H_{1}$ is the standard model like Higgs and $H_{2}$ is the triplet like Higgs. The mixing angle is given by

$$
\tan 2 \theta_{0}=\frac{\mu v}{\left(M_{\Delta}^{2}-M_{H}^{2}\right)} .
$$

The corresponding mass eigenvalues are $M_{H_{1}}$ (SM Higgs like) and $M_{H_{2}}$ (triplet like) and are given as :

$$
\begin{gathered}
M_{H_{1}}^{2} \approx M_{H}^{2}-\frac{(\mu v / 2)^{2}}{M_{\Delta}^{2}-M_{H}^{2}} \\
M_{H_{2}}^{2} \approx M_{\Delta}^{2}+\frac{(\mu v / 2)^{2}}{M_{\Delta}^{2}-M_{H}^{2}} .
\end{gathered}
$$

Since the addition of a scalar triplet can modify the $\rho$ parameter, which is not differing from SM value: $\rho=$ $1.00037 \pm 0.00023\left[29\right.$, so we have a constraint on the vev $u_{\Delta}$ as:

$$
u_{\Delta} \leq 3.64 \mathrm{GeV} .
$$

For different values of $M_{\Delta}$ we have shown $\mu$ as a function of $\sin \theta_{0}$ in Fig. (1). From Eqs. (20), (4) and (22) we see

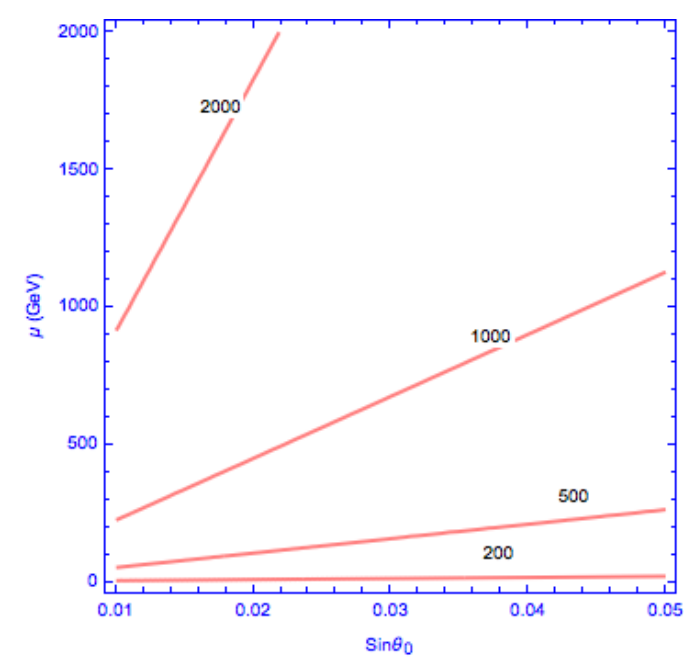

FIG. 1: Contours of different values of $M_{\Delta}$ (in $\mathrm{GeV}$ ) in the plane of $\mu$ versus $\sin \theta_{0}$

that there exist an upper bound on the mixing angle

$$
\sin \theta_{0}<0.02\left(\frac{174 \mathrm{GeV}}{v}\right)\left(\frac{1}{1-0.39 \frac{\left(M_{H} / 125 \mathrm{GeV}\right)^{2}}{\left(M_{\Delta} / 200 \mathrm{GeV}\right)^{2}}}\right)
$$


We also get a constraint on $\sin \theta_{0}$ from the decay of SM Higgs branching fractions for different channels. For example let us take the decay of $H_{1}$ to $\tau$ leptons. The decay width is given by :

$$
\Gamma=\frac{M_{H_{1}}}{8 \pi} \frac{m_{\tau}^{2}}{v^{2}}\left(1-\frac{4 m_{\tau}^{2}}{M_{H_{1}}^{2}}\right)^{3 / 2}\left(1-\sin ^{2} \theta_{0}\right)
$$

Comparing with the experimental branching fraction $\operatorname{Br}\left(H_{1} \rightarrow \tau \tau\right)=6.272 \times 10^{-2}$, we found that $\sin \theta_{0}=0.176$. So any value less than this will be allowed by the experiment. Thus we see that the bound obtained on the mixing angle is less constraining than the bound from $\rho$ parameter. Therefore, we will use the constraint on the mixing angle, obtained from $\rho$ parameter, while calculating the DM-nucleon elastic scattering in section (VI). Since the doublet-triplet scalar mixing is found to be small we assume that the flavour eigenstates are the mass eigenstates and treat $M_{H_{1}}=M_{H}, M_{H_{2}}=M_{\Delta}$ through out the calculation.

We assume that there is no mixing between the neutral CP-odd states as well as in the charged states. So that the $\xi^{0}$ is absorbed in the unitary gauge by the gauge bosons after the symmetry breaking and the charged triplet scalar fields will remain as the mass eigen fields.

\section{NON ZERO NEUTRINO MASS}

The coupling of $\Delta$ to lepton and Higgs doublets combinely break the lepton number by two units as given in Eq. (5). As a result the $\Delta L_{\alpha} L_{\beta}$ coupling gives Majorana masses to three flavor of active neutrinos as:

$$
\left(M_{\nu}\right)_{\alpha \beta}=\sqrt{2}\left(f_{L}\right)_{\alpha \beta}\langle\Delta\rangle=\left(f_{L}\right)_{\alpha \beta} \frac{-\mu v^{2}}{\sqrt{2} M_{\Delta}^{2}} .
$$

Taking $\mu \simeq M_{\Delta} \simeq \mathcal{O}\left(10^{14}\right) \mathrm{GeV}$, we can explain neutrino masses of order $0.1 \mathrm{eV}$ with a coupling strength $f_{L}=1$. However, the scale of $M_{\Delta}$ can be brought down to TeV scales by taking the smaller couplings.

To get the neutrino mass eigen values, the above mass matrix can be diagonalised by the usual $U_{P M N S}$ matrix as :

$$
M_{\nu}=U_{\mathrm{PMNS}} M_{\nu}^{\text {diag }} U_{\mathrm{PMNS}}^{T},
$$

where $U_{P M N S}$ is given by

$$
U_{\mathrm{P} M N S}=\left(\begin{array}{ccc}
c_{12} c_{13} & s_{12} c_{13} & s_{13} e^{-i \delta_{13}} \\
-s_{12} c_{23}-c_{12} s_{23} s_{13} e^{i \delta_{13}} & c_{12} c_{23}-s_{12} s_{23} s_{13} e^{i \delta_{13}} & s_{23} c_{13} \\
s_{12} s_{23}-c_{12} c_{23} s_{13} e^{i \delta_{13}} & -c_{12} s_{23}-s_{12} c_{23} s_{13} e^{i \delta_{13}} & c_{23} c_{13}
\end{array}\right) \cdot U_{p h},
$$

with $c_{i j}, s_{i j}$ stand for $\cos \theta_{i j}$ and $\sin \theta_{i j}$ respectively and $U_{p h}$ is given by

$$
U_{p h}=\operatorname{Diag}\left(e^{-i \gamma_{1}}, e^{-i \gamma_{2}}, 1\right) .
$$

Where $\gamma_{1}, \gamma_{2}$ are two Majorana phases.

\section{RELIC ABUNDANCE OF DM}

The lightest particle $N_{1}$, which is stabilized by the imposed $Z_{2}$ symmetry, serves as a viable candidate of DM. The relic abundance of $N_{1}$ can be obtained through its interaction with $N^{-}$and $N_{2}$. The main processes which contribute to the relic abundance of DM without involving triplet scalar are [14] :

$N_{1} \overline{N_{1}} \rightarrow H H, Z H, W^{+} W^{-}, Z Z, f \bar{f}$

$N_{1} \overline{N_{2}} \rightarrow H H, Z H, W^{+} W^{-}, Z Z, f \bar{f}$

$N_{2} \overline{N_{2}} \rightarrow H H, Z H, W^{+} W^{-}, Z Z, f \bar{f}$

$N_{1} N^{ \pm} \rightarrow W^{ \pm} \gamma, W^{ \pm} H, W^{ \pm} Z, f^{\prime} \bar{f}$

$N_{2} N^{ \pm} \rightarrow W^{ \pm} \gamma, W^{ \pm} H, W^{ \pm} Z, f^{\prime} \bar{f}$

$N^{ \pm} N^{ \pm} \rightarrow W^{ \pm} W^{ \pm}, Z H, \gamma Z, \gamma \gamma, Z Z, f \bar{f}$

In presence of the light scalar triplet $\Delta$, there will be additional s-channel processes through $\Delta^{0}$ mediation as well as processes involving $\Delta$ particles in the final states. The relevant processes are : 


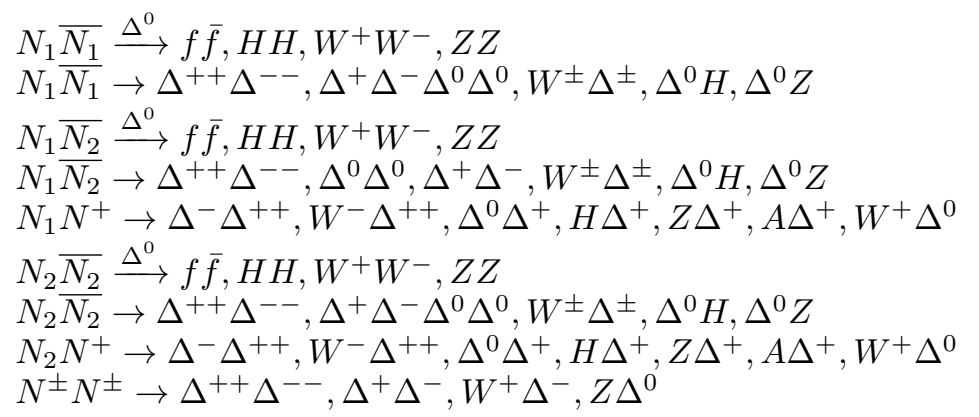

Relic density for $N_{1}$ is given by [30]

$$
\Omega_{N_{1}} h^{2}=\frac{1.09 \times 10^{9} \mathrm{Gev}^{-1}}{g_{\star}^{1 / 2} m_{\mathrm{pl}}} \frac{1}{J\left(x_{f}\right)},
$$

where $J\left(x_{f}\right)$ is given by

$$
J\left(x_{f}\right)=\int_{x_{f}}^{\infty} \frac{\langle\sigma|v|\rangle_{\mathrm{eff}}}{x^{2}} d x
$$

where $\langle\sigma|v|\rangle_{\text {eff }}$ is thermal average of annihilation and coannihilation cross-sections of the DM particle. The expression for effective cross-section can be written as :

$$
\begin{aligned}
\langle\sigma|v|\rangle_{\mathrm{eff}}= & \frac{g_{1}^{2}}{g_{\mathrm{eff}}^{2}} \sigma\left(N_{1} N_{1}\right)+2 \frac{g_{1} g_{2}}{g_{\mathrm{eff}}^{2}} \sigma\left(N_{1} N_{2}\right)(1+\omega)^{3 / 2} \exp (-x \omega) \\
& +2 \frac{g_{1} g_{3}}{g_{\mathrm{eff}}^{2}} \sigma\left(N_{1} N^{-}\right)(1+\omega)^{3 / 2} \exp (-x \omega) \\
& +2 \frac{g_{2} g_{3}}{g_{\mathrm{eff}}^{2}} \sigma\left(N_{2} N^{-}\right)(1+\omega)^{3} \exp (-2 x \omega)+\frac{g_{2}^{2}}{g_{\mathrm{eff}}^{2}} \sigma\left(N_{2} N_{2}\right)(1+\omega)^{3} \exp (-2 x \omega) \\
& +\frac{g_{3}^{2}}{g_{\mathrm{eff}}^{2}} \sigma\left(N^{-} N^{-}\right)(1+\omega)^{3} \exp (-2 x \omega) .
\end{aligned}
$$

In this equation $g_{1}, g_{2}, g_{3}$ represent spin degrees of freedom for particles $N_{1}, N_{2}, N^{-}$respectively and their values are 2 for all. $\omega$ stands for the mass splitting ratio, given by $\omega=\frac{M_{i}-M_{1}}{M_{1}}$, where $M_{i}$ is the mass of $N_{2}$ and $N^{ \pm}$. The effective degrees of freedom denoted by $g_{\text {eff }}$, and is given by

$$
g_{\mathrm{eff}}=g_{1}+g_{2}(1+\omega)^{3 / 2} \exp (-x \omega)+g_{3}(1+\omega)^{3 / 2} \exp (-x \omega)
$$

To calculate the relic density we use the code micrOMEGAs [31]. We have plotted in fig. 2 the relic density of DM as a function of its mass keeping the mass difference $M_{2}-M_{1}=500 \mathrm{GeV}$, for three different values of the mixing angle: $\sin \theta=0.1,0.2,0.3$, shown as red, green, purple respectively in the plot. In the left panel of the fig. 2 we use $M_{\Delta}=200 \mathrm{GeV}$, whereas in the right panel of fig. 2 we use $M_{\Delta}=1000 \mathrm{GeV}$. The black horizontal line corresponds to the observed relic density: $\Omega_{\mathrm{DM}} h^{2}=0.1199 \pm 0.0027$ by PLANCK [4]. From fig. 2, we notice that there is a sharp decrease in relic density near three different points. These three points correspond to the resonant annihilation of DM to the SM particles via the s- channel processes mediated by $Z, \mathrm{~h}$ and $\Delta^{0}$. From these figures it is clear that as $\sin \theta$ increases relic density decreases. It is due to the fact that the $Z$ and $\Delta$ mediated cross-section increases for increase in $\sin \theta$, and hence yield a low relic density. For both the plots in fig 2 we fix the ratio of Majorana couplings to be: $\frac{f_{L}}{f_{N}}=10^{-2}$. From the plots in the fig. 22 we conclude that the $\Delta$ field is contributing to the relic density only near the resonance points. Therefore, we can not expect any change in the parameter space if we vary the ratio of Majorana couplings: $\frac{f_{L}}{f_{N}}$. The cross-sections involving the scalar triplet in the final states does not affect the relic abundance since in this region of parameter space $\left(M_{1}>M_{\Delta}\right)$ the cross-sections involving gauge bosons in the final state dominate. As the mass splitting between $N_{1}$ and $N_{2}$ is taken to be very large in the above cases, only annihilation channels contribute. Coannihilation channels are Boltzmann suppressed due to this large mass splitting. We can also see that the triplet effect is also reduced when its mass is $1000 \mathrm{GeV}$. This is because the total cross-section is dominated by $N_{1} \bar{N}_{1} \rightarrow W^{+} W-$ and the delta mediated s-channel contribution is suppressed due to the large triplet scalar mass present in the propagator. 

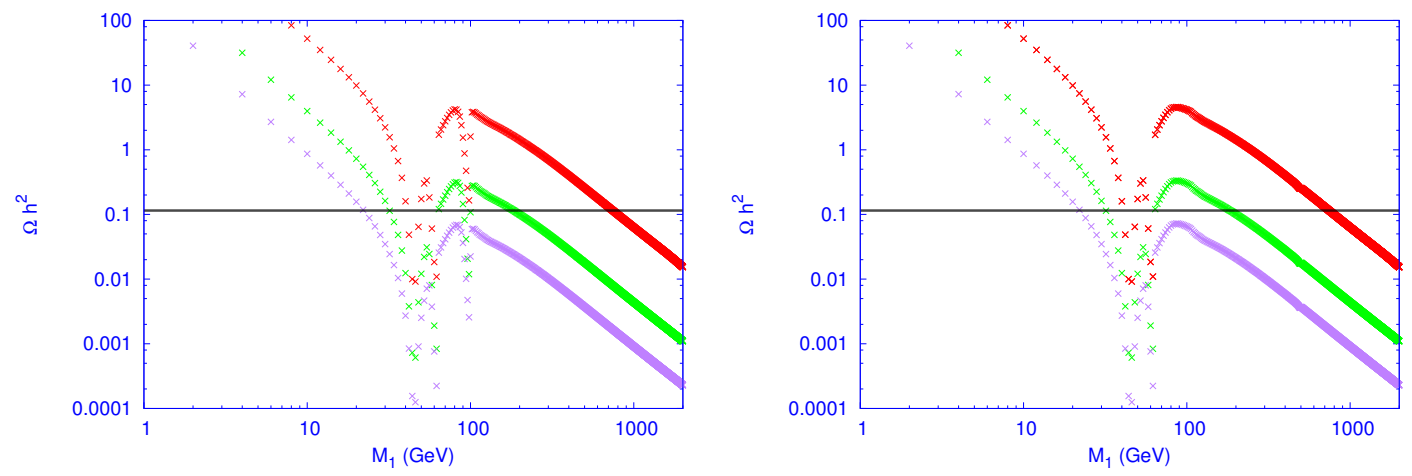

FIG. 2: Relic density of DM as a function of its mass $M_{1}$ for different values of $\sin \theta=0.1,0.2,0.3$, shown by red, green and purple respectively. The value triplet mass: $M_{\Delta}=200,1000 \mathrm{GeV}$ is fixed respectively for left and right panel. All these plots are generated keeping a fixed value of the mass splitting $M_{2}-M_{1}=500 \mathrm{GeV}$.
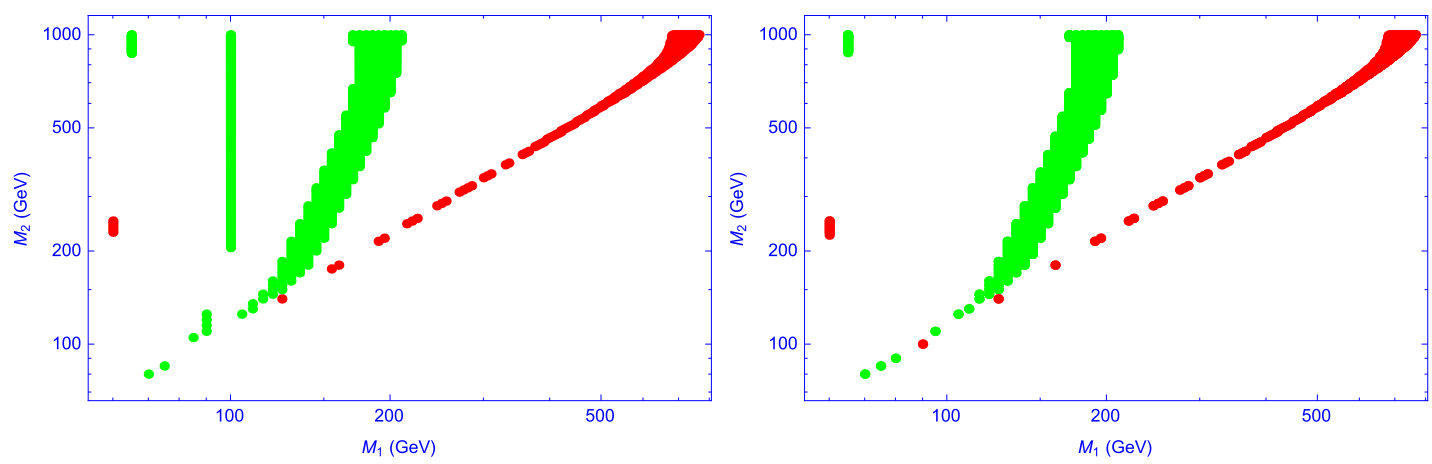

FIG. 3: Scatter plot for correct relic density, shown by red and green colored points for $\sin \theta=0.1,0.2$ respectively in the plane of $M_{1}$ and $M_{2}$. The triplet mass $M_{\Delta}=200,1000 \mathrm{GeV}$ is taken respectively for the left and right panel plots. We fixed the value of Majoranan coupling: $f_{L} / f_{N}=10^{-2}$ in both the figures.

Now we will try to show the effect of mass-splitting between $N_{1}$ and $N_{2}$ on dark matter relic density. For this, in fig. 3, we have shown a scatter plot for correct relic density in the plane of $M_{1}$ and $M_{2}$. Red and green points satisfy the constraint of relic density for $\sin \theta=0.1,0.2$ respectively. Let us first consider the green points satisfying the relic density. The points on the vertical bar are coming from annihilation channels. In this region of parameter space the coannihilation cross-sections are Boltzmann suppressed due to large mass splitting. As the mass splitting decreases the co-annihilation channels also contribute equally as the annihilation channels. For singlet-doublet fermion mixing: $\sin \theta=0.1$ (Red points), we get the correct relic density for DM mass all the way to $800 \mathrm{GeV}$. However, as the mixing increases, say $\sin \theta=0.2$ (Green points), large contribution from $N_{1} \bar{N}_{1} \rightarrow W^{+} W^{-}$dominates and hence we get a limited parameter space for correct relic density in the low mass region by keeping large mass splitting between $N_{1}$ and $N_{2}$. For the same reasosn, we don't get any parameter space which satisfy the correct relic density with further increase of singlet-doublet mixing. The top left points, near DM mass $100 \mathrm{GeV}$ in fig 3 are due to the $\Delta^{0}$ mediated s- channel annihilation processes because the cross-section is exactly the required one for relic density. We are not getting any such points for $M_{\Delta}=1000 \mathrm{GeV}$, because the cross-section is not meeting the relic density criteria at the resonance point which can be clearly seen from fig. 2

The $\Delta M$ dependency on the relic density for a specific choice of mixing angle is shown in Fig. 4. In the left panel we use $\sin \theta=0.1$ and that in the right panel $\sin \theta=0.0001$. We plot different slices with constant $\Delta M=10,20,30,40,100$ $\mathrm{GeV}$ as shown in Blue, Green, Orange, Purple, Red respectively. We note here that with larger $\Delta M$, the annihilation cross-section increases due to enhancement in Yukawa coupling $Y \propto \Delta M$. However, co-annihilation decreases due to increase in $\Delta M$ as $\sigma \propto e^{-\Delta M}$. Note that in the small $\sin \theta$ limit the dominant contribution to relic density comes from the channels involving only $N_{2}$ and $N^{ \pm}$in the initial state going to SM gauge bosons, as mentioned in the beginning of this section. The processes involving $N_{1} N_{1} \rightarrow$ SM particle are heavily suppressed with small $\sin \theta$. As a result, we first get relics of $N_{2}$ and $N^{-}$which subsequently decay to $N_{1}$ before $N_{1}$ freezes out. In particular, if the mass splitting between $N^{-}$and $N_{1}$ is more than $80 \mathrm{GeV}$, then $N^{-}$decays through two body process: $N^{-} \rightarrow N_{1}+W^{-}$. 

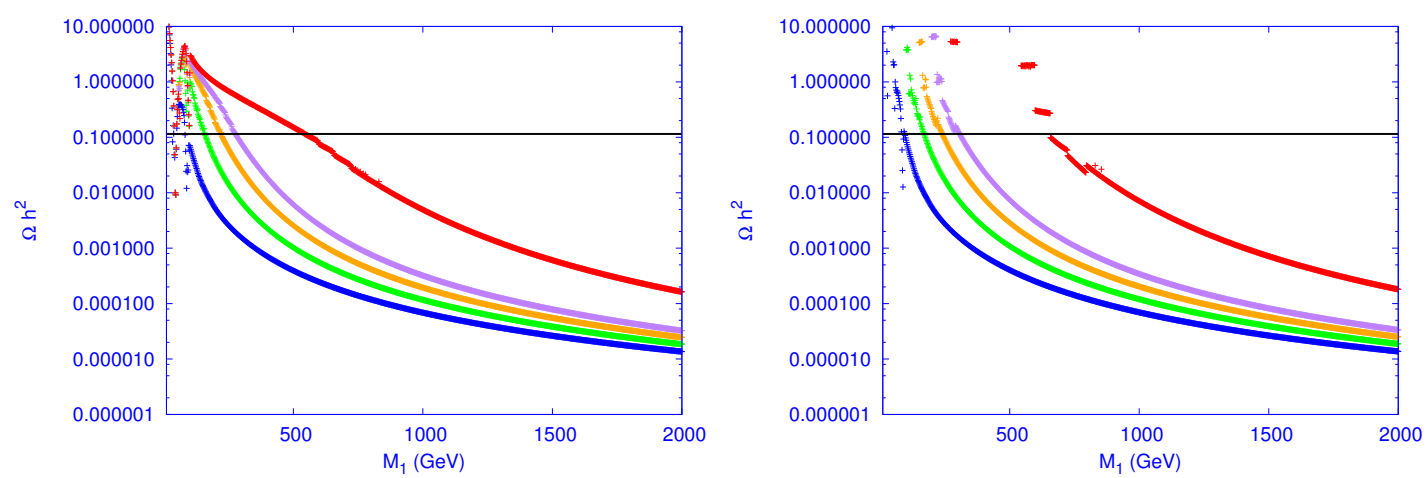

FIG. 4: Left : $\Omega$ versus DM mass $M_{D M}$ in $\mathrm{GeV}$ for $\sin \theta=0.1$ and $\Delta M=10,20,30,40,100$ (Blue, Green, Orange, Purple, Red respectively). Right : $\Omega$ versus DM mass $M_{D M}$ in $\mathrm{GeV}$ for $\sin \theta=0.0001$ and $\Delta M=10,20,30,40,100$ (Blue, Green, Orange, Purple, Red respectively). Horizontal line shows the correct relic density. We fixed the value $f_{L} / f_{N}=10^{-2}$ for all the plots.

However, if the mass splitting between $N^{-}$and $N_{1}$ is less than $80 \mathrm{GeV}$, than the former decays through the three body process, say $N^{-} \rightarrow N_{1}+\ell^{-}+\overline{\nu_{\ell}}$. Notice that the mixing angles $\sin \theta=0.1,0.0001$ used simultaneously in the left and right-panel of Fig. (4) are much larger than the lower bound obtained on the singlet-doublet fermoin mixing angle as given in eq. 16 by considering the 3-body decay of $N^{-}$, namely $\sin \theta>\mathcal{O}\left(10^{-5}\right)$.

For large $\Delta M$ the co-annihilation cross-sections decrease, which are expected to be dominant processes in the small $\sin \theta$ limit. As a result the relic abundance increases for a particular value of $M_{1}$. Hence we need a larger $\Delta M$ for larger DM mass so that the relic density will be in the observed limit.

\section{DIRECT DETECTION OF DM THROUGH INELASTIC SCATTERING WITH THE NUCLEI}

The Lagrangian relevant to kinetic, mass terms and interaction with the $Z$ boson of the DM can be rewritten as :

$$
\begin{aligned}
\mathcal{L}_{D M} \supset & i \overline{N_{1}}\left(\not \partial+i g_{z} Z_{\mu} \gamma^{\mu}\right) N_{1} \\
& -M_{1} \overline{N_{1}} N_{1}-\frac{1}{2} f_{N}\left(\overline{N_{1}^{C}} P_{L} N_{1}+\text { h.c }\right) \Delta^{0}-\frac{1}{2} f_{N}\left(\overline{N_{1}^{C}} P_{R} N_{1}+\text { h.c }\right) \Delta^{0},
\end{aligned}
$$

where $g_{z}$ is the coupling strength with $Z$ boson and $M_{1}$ can be identified as Dirac mass for DM. When $\Delta$ gets a vev, the DM gets small Majorana mass $m=f_{N} u_{\Delta}$. The mass matrix is written as :

$$
M_{D M}=\left(\begin{array}{cc}
m & M_{1} \\
M_{1} & m
\end{array}\right)
$$

The presence of small Majorana mass terms for the DM split the Dirac state into two Majorana states $N_{1}^{\prime}$ and $N_{2}^{\prime}$. The mass eigen values are :

$$
M_{1,2}^{\prime}=M_{1} \pm m
$$

and the mixing angle is $\eta=\pi / 4$ which is the maximal mixing. The Lagrangian with gauge field $Z$ in terms of the new eigenstates is given as

$$
\mathcal{L}_{D M} \supset \frac{1}{2} \overline{N_{1}^{\prime}} i \gamma^{\mu} \partial_{\mu} N_{1}^{\prime}-\frac{1}{2} M_{1}^{\prime} \overline{N_{1}^{\prime}} N_{1}^{\prime}+\frac{1}{2} \overline{N_{2}^{\prime}} i \gamma^{\mu} \partial_{\mu} N_{2}^{\prime}-\frac{1}{2} M_{2}^{\prime} \overline{N_{2}^{\prime}} N_{2}^{\prime}+i g_{z} \overline{N_{2}^{\prime}} \gamma^{\mu} N_{1}^{\prime} Z_{\mu},
$$

From the above expression the dominant gauge interaction is off-diagonal, and the diagonal interaction vanishes. As a result there will be inelastic scattering possible for the DM with the nucleus. The mass splitting between the two mass eigen states is given by:

$$
\delta M=M_{2}^{\prime}-M_{1}^{\prime}=2 m=f_{N} u_{\Delta} .
$$

In this case, the minimum velocity of the DM needed to register a recoil inside the detector is given by [32 36] :

$$
v_{\min }=c \sqrt{\frac{1}{2 m_{n} E_{R}}}\left(\frac{m_{n} E_{R}}{\mu_{r}}+\delta M\right),
$$


where $E_{R}$ is the recoil energy of the nucleon and $\mu_{r}$ is the reduced mass. If the mass splitting is above a few hundred $\mathrm{keV}$, then it will be difficult to excite $N_{2}^{\prime}$ with the largest possible kinetic energy of the DM. So the inelastic scattering mediated by Z-boson will be forbidden. As a result the constraints coming from direct detection can be relaxed. This in an important consequence in presence of the scalar triplet $\Delta$.

\section{DIRECT DETECTION OF DM THROUGH ELASTIC SCATTERING WITH THE NUCLEI}

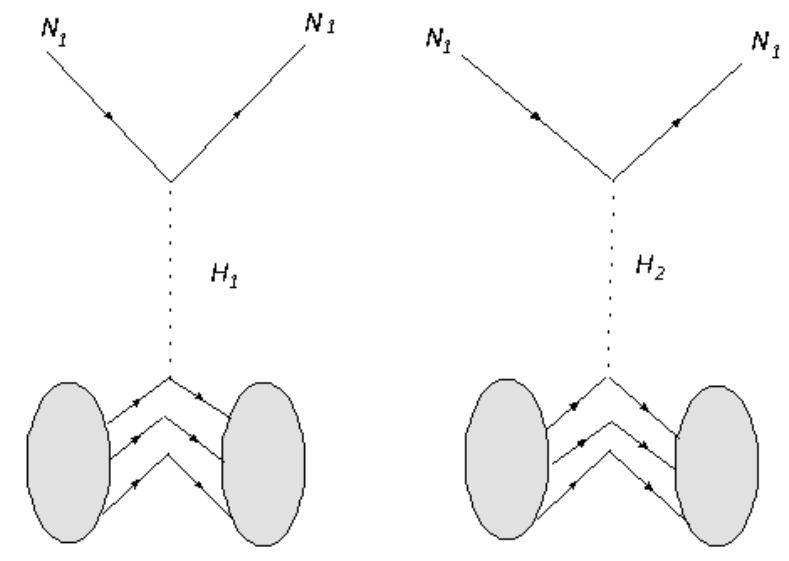

FIG. 5: Feynman diagrams for direct detection of $N_{1}$ DM via Higgs mediation.

We shall now point out constraints on the model parameters from direct search of DM via Higgs mediation. The relevant diagrams through which $N_{1}$ interacts with the nuclei are shown in Fig. (5). In particular, our focus will be on Xenon-100 [26] and LUX [27] which at present give strongest constraint on spin-independent DM-nucleon cross-section from the null detection of DM yet. In our model, this in turn puts a stringent constraint on the singlet-doublet mixing angle $\sin \theta$ for spin independent DM-nucleon interaction mediated via the $H_{1}$ and $H_{2}$-bosons (see in the Fig. (5p). The cross-section per nucleon is given by [37, 38,

$$
\sigma_{\mathrm{SI}}=\frac{1}{\pi A^{2}} \mu_{r}^{2}|\mathcal{M}|^{2}
$$

where $A$ is the mass number of the target nucleus, $\mu_{r}=M_{1} m_{n} /\left(M_{1}+m_{n}\right) \approx m_{n}$ is the reduced mass, $m_{n}$ is the mass of nucleon (proton or neutron) and $\mathcal{M}$ is the amplitude for DM-nucleon cross-section. There are two t-channel processes through which DM can interact with the nucleus which is shown in the fig 5 The amplitude is given by:

$$
\mathcal{M}=\sum_{i=1,2}\left[Z f_{p}^{i}+(A-Z) f_{n}^{i}\right]
$$

where the effective interaction strengths of DM with proton and neutron are given by:

$$
f_{p, n}^{i}=\sum_{q=u, d, s} f_{T q}^{(p . n)} \alpha_{q}^{i} \frac{m_{(p, n)}}{m_{q}}+\frac{2}{27} f_{T G}^{(p, n)} \sum_{q=c, t, b} \alpha_{q}^{i} \frac{m_{p . n}}{m_{q}}
$$

with

$$
\begin{gathered}
\alpha_{q}^{1}=\frac{Y \sin 2 \theta \cos ^{2} \theta_{0}}{M_{H}^{2}}\left(\frac{m_{q}}{v}\right) \\
\alpha_{q}^{2}=-\frac{Y \sin 2 \theta \sin ^{2} \theta_{0}}{M_{\Delta}^{2}}\left(\frac{m_{q}}{v}\right) .
\end{gathered}
$$

In Eq. 41, the different coupling strengths between DM and light quarks are given by [1] $f_{T u}^{(p)}=0.020 \pm 0.004$, $f_{T d}^{(p)}=0.026 \pm 0.005, f_{T s}^{(p)}=0.118 \pm 0.062, f_{T u}^{(n)}=0.014 \pm 0.004, f_{T d}^{(n)}=0.036 \pm 0.008, f_{T s}^{(n)}=0.118 \pm 0.062$. The coupling 
of DM with the gluons in target nuclei is parameterized by

$$
f_{T G}^{(p, n)}=1-\sum_{q=u, d, s} f_{T q}^{(p, n)}
$$

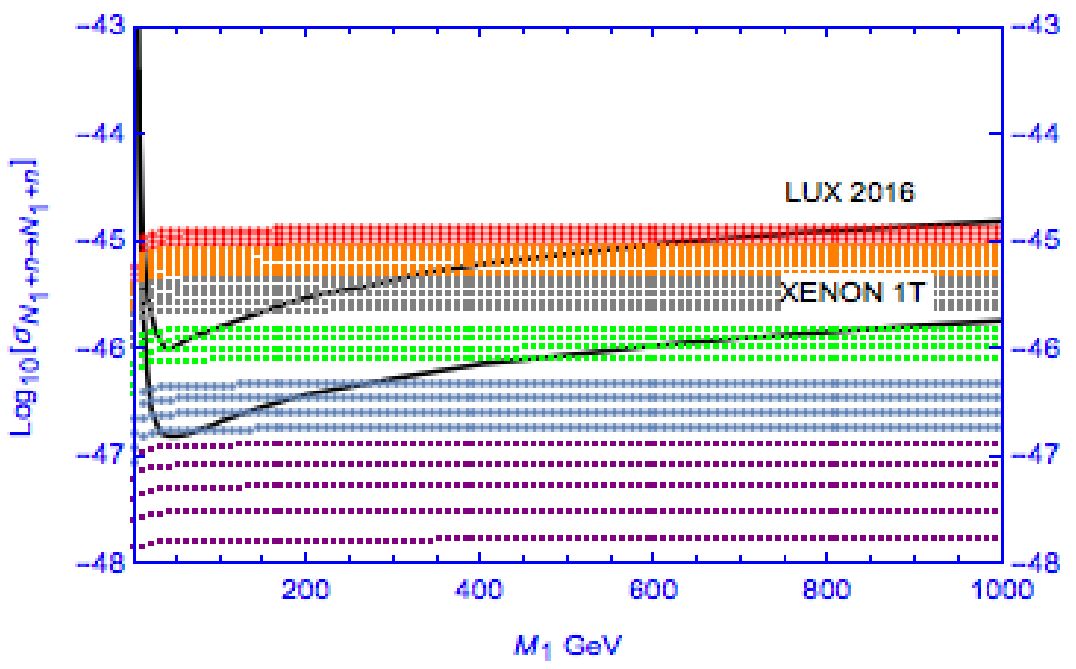

FIG. 6: Spin Independent direct detection cross-section for DM as a function of DM mass for $\sin \theta=\{0.05-0.1\}$ (Purple), $\sin \theta=\{0.1-0.15\}$ (Lilac), $\sin \theta=\{0.15-0.2\}$ (Green), $\sin \theta=\{0.2-0.25\}$ (Gray), $\sin \theta=\{0.25-0.3\}$ (Orange), $\sin \theta=\{0.3-0.35\}$ (Red). Black curves are the data from LUX and XENON $1 \mathrm{~T}$ prediction. Value of $\Delta M=100 \mathrm{GeV}$ is fixed while $M_{\Delta}=200 \mathrm{GeV}$ is used. The scalar mixing angle $\sin \theta_{0}=0.05$ is fixed for the calculation.

We have plotted the spin independent direct detection cross-section as a function of DM mass in the Fig 6 by taking the value of $M_{\Delta}=200 \mathrm{GeV}$. The plot is generated using different values of the singlet-doublet mixing angle: $\sin \theta=\{0.05-0.1\}$ (Purple), $\sin \theta=\{0.1-0.15\}$ (Lilac), $\sin \theta=\{0.15-0.2\}$ (Green), $\sin \theta=\{0.2-0.25\}$ (Gray), $\sin \theta=\{0.25$ $0.3\}$ (Orange) $\sin \theta=\{0.3-0.35\}$ (Red). Two Black lines are drawn in the same figure, which shows the experimental limit on the SI nuclei-DM cross-section with DM mass as predicted from LUX 2016 and XENON 1T. We see that the constraint from XENON 100 is very loose on the parameter space.

Since the mixing between $\Delta-h$ is small: $\sin \theta_{0}<5 \times 10^{-2}$, the contribution to the cross-section by the $H_{2}$ mediated diagram is suppressed. This is also further suppressed by the large mass of $M_{\Delta}$ present in the propagator. For this reason we will not getting any difference in cross-section for the higher values of $M_{\Delta}$ as the cross-section is dominated by $H_{1}$ mediation only. It is also noticed from the figure that most of the parameter space is allowed for $\sin \theta \leq 0.15$ except for small values of DM mass.

\section{DECAY OF $N^{-}$AND THE DISPLACED VERTEX SIGNATURE}

The phenomenology of the charged partner of DM is quite interesting. If the mass splitting between $N^{ \pm}$and $N_{1}$ is less than mass of $W^{-}$, then $N^{-}$will decay via three body suppressed process: $N^{-} \rightarrow N_{1} \ell \nu_{\ell}$ and $N^{-} \rightarrow N_{1}+$ di - jets. The relevant Feynman diagrams for the decay is shown in Fig. 7. However the right figure is suppressed due to the small coupling of triplet $\Delta$ with the leptons and the large mass $M_{\Delta}$ present in the propagator. So the dominant contribution for decay of $N^{-}$is coming from the left diagram of Fig. 7. The decay rate for the process $N^{-} \rightarrow N_{1} \ell \nu_{\ell}$ is given in Eq12.

In the left panel of Fig. 8, a scatter plot is shown taking relic abundance as a function of DM mass keeping the mass splitting less than $50 \mathrm{GeV}$. We have also shown the correct relic abundance as allowed by the PLANCK data with a horizontal solid black line. We choose those set of points from the relic abundance data which will be allowed by the PLANCK result and use them to calculate the displaced vertex signature of $N^{ \pm}$in the right-panel of Fig. (8). We observe that the displaced vertex becomes very small for larger values of $M^{ \pm}$, as the inverse of decay width $\Gamma^{-1}$ is inversely proportional to the mass of decaying charged particle. In Fig. 8, we fix the singlet-doublet mixing angle to be $\sin \theta=3 \times 10^{-4}$. The important point to be noted here is that to get a large displaced vertex we need a small 

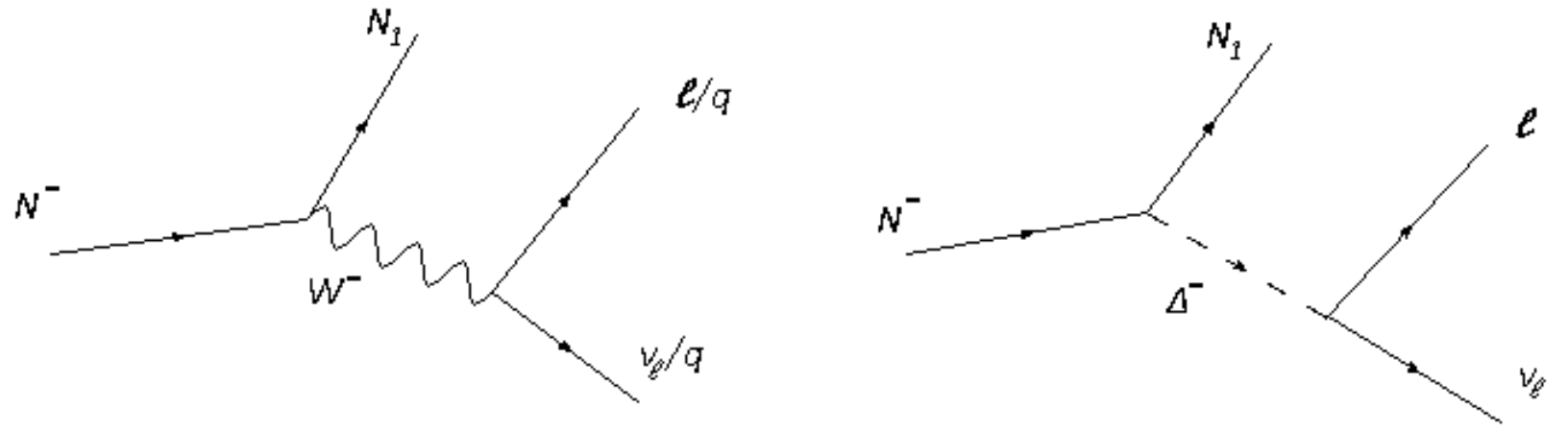

FIG. 7: Feynman diagrams for 3 body decay of $N^{-}$.
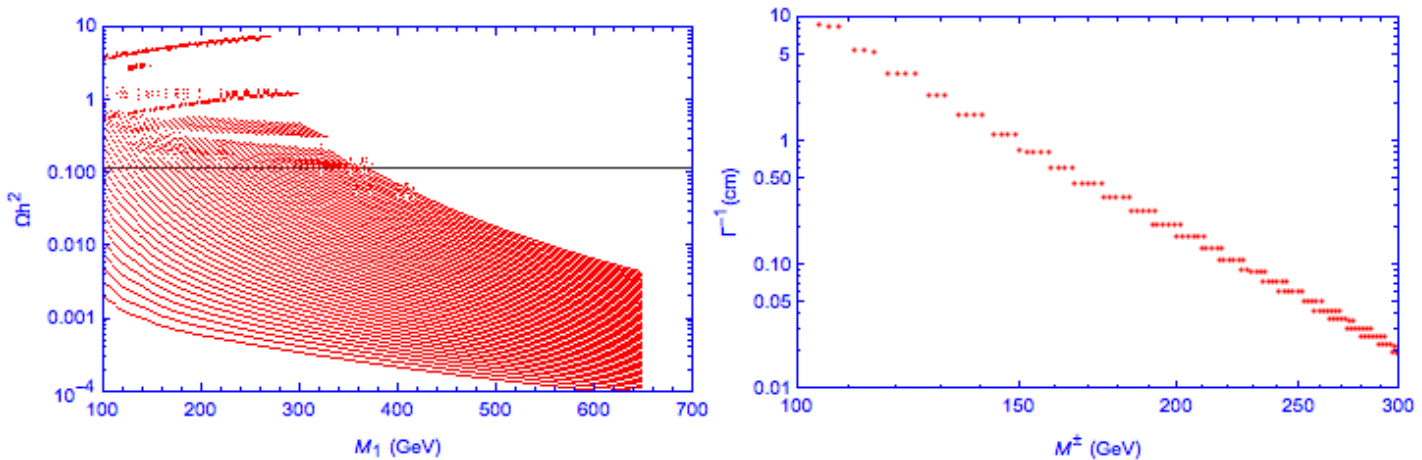

FIG. 8: Left panel: Scatter plot showing relic abundance as a function of DM mass with mass splitting less than 50 GeV. Black solid line shows the correct relic abundance as allowed by PLANCK data. Right panel: Displaced vertex as a function of $M^{ \pm}$. Value of mixing angle $\sin \theta=3 \times 10^{-4}$ is used for calculation.

mixing angle between the singlet and doublet. In fact, the small mixing angle is favored by all the constraints we discussed in previous sections, such as correct relic abundance and null detection of DM at direct search experiments. However, from Eq. (16) we also learnt that the singlet-doublet mixing can not be arbitrarily small and therefore, the displaced vertex can not be too large.

\section{SUMMARY AND CONCLUSION}

We explored the possibility of a singlet-doublet mixed vector-like fermion dark matter in presence of a scalar triplet. The mixing angle: $\sin \theta$ between the singlet and doublet plays an important role in the calculation of relic abundance as well as direct detection. We found that the constraint from null detection of DM at direct search experiments and relic abundnace can be satisfied in a large region of parameter space for small mixing angle: $\sin \theta \leq 0.2$. If the scalar triplet is light, say $M_{\Delta} \lesssim 500 \mathrm{GeV}$, then it contributes to relic abundance only near the resonance. On the other hand, if $M_{\Delta} \gtrsim 1 \mathrm{TeV}$, then it decouples and hence does not contribute to relic abundance of DM.

The scalar triplet couples symmetrically to lepton doublets as well as to the doublet component of the DM. Therefore, when the scalar triplet acquires an induced vev, it not only gives Majorana masses to the light neutrinos but also induce a sub-GeV Majorana mass to the DM. As a result the DM, which was originally a vector-like Dirac fermion splits into two pseudo-Dirac fermions with a mass separation of sub-GeV order. Due to this reason the Z-mediated inelastic scattering of the DM with nucleon is suppressed. However, we found that the spin independent direct detection of DM through the SM Higgs mediation is in the right ballpark of Xenon-1T. The absence of $Z$ mediated DM-nucleon cross-section relaxes the constraint on mixing angle $\sin \theta$ as we can go as high as $\sin \theta=0.3$ for DM mass $M_{1}>400 \mathrm{GeV}$. But this high value of $\sin \theta$ is not allowed by the correct relic abundance. So the bound on spin independent direct detection cross-section does not put stronger constraint on the mixing angle than we got 
from relic abundance.

The $\rho$ parameter in the SM restricts the vev of scalar triplet to $u_{\Delta} \leq 3.64 \mathrm{GeV}$. This in turn gives the mixing between the SM Higgs and $\Delta$ to be $\sin \theta_{0} \mathcal{O}\left(10^{-2}\right)$ even if the $M_{\Delta} \lesssim 500 \mathrm{GeV}$. Therefore, $\Delta$ does not contribute significantly to the spin independent direct detection cross-section.

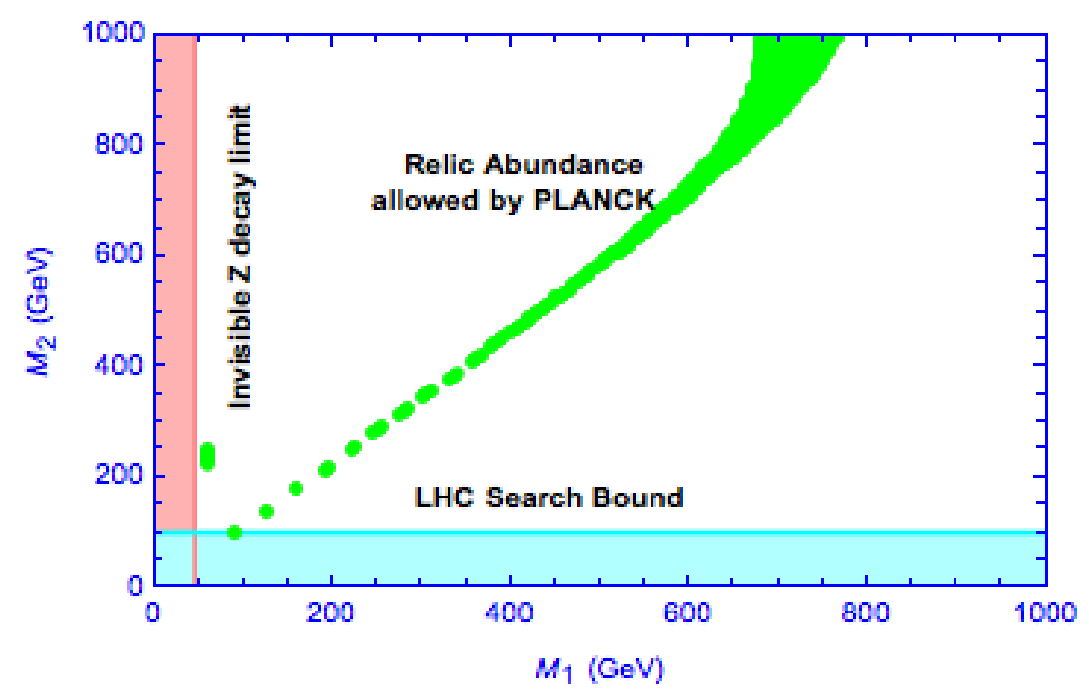

FIG. 9: Summary of all constraints in the plane of $M_{1}-M_{2}$ using $\sin \theta=0.1$.

We summarize the constraints on the parameters in Fig. 9, where we have shown the allowed values in the plane of $M_{1}-M_{2}$ using $\sin \theta=0.1$. The green points are allowed by the relic abundance of DM by taking the constraint from PLANCK data. However there are small regions which are disfavoured by various experimental searches. For example, the region in cyan color is disfavoured by the collider search of $N^{ \pm}$and hence the allowed values are given by $M^{ \pm} \sim M_{2}>100 \mathrm{GeV}$. The mass of $N_{1}$ (DM), i.e., $M_{1}>45 \mathrm{GeV}$, is required in order to relax the severe constraints from the invisible $Z$ boson decay [14. It is important to note that the direct search of DM does not give any constraint on the parameter space spanned by $M_{1}-M_{2}$ for $\sin \theta=0.1$.

The charged partner of the DM gives interesting signatures at colliders if $M^{ \pm}-M_{1} \lesssim 80 \mathrm{GeV}$. As a result the two body decay of $N^{ \pm}$is forbidden. The only way it can decay is the three body decay. For example, the notable one is $N^{-} \rightarrow N_{1} \ell^{-} \overline{\nu_{\ell}}$. In the small singlet-doublet mixing limit we get a displaced vertex of $10 \mathrm{~cm}$ for $M^{ \pm} \sim 100 \mathrm{GeV}$ and a mass splitting of few tens of $\mathrm{GeV}$ while satisfying the constraint from observed relic abundance.

\section{Acknowledgement}

SB would like to acknowledge the DST-INSPIRE project with grant no PHY/P/SUB/01 at IIT Guwahati. 
[1] G. Bertone, D. Hooper and J. Silk, Particle Dark Matter: Evidence, Candidates and Constraints, Phys. Rept. 405 , 279 (2005), arXiv:hep-ph/0404175

[2] G. Jungman, M. Kamionkowski and K. Griest, Supersymmetric Dark Matter, Phys. Rept. 267, 195 (1996), arXiv:hep$\mathrm{ph} / 9506380$

[3] G. Hinshaw et al. [WMAP Collaboration], Astrophys. J. Suppl. 208, 19 (2013) arXiv:1212.5226 [astro-ph.CO]].

[4] P. A. R. Ade et al. [Planck Collaboration], Astron. Astrophys. 571, A16 (2014), arXiv:1303.5076 [astro-ph.CO].

[5] S. Fukuda et al. [Super-Kamiokande Collaboration], Phys. Rev. Lett. 86, 5656 (2001) hep-ex/0103033.

[6] S. M. Bilenky, J. Hosek and S. T. Petcov, Phys. Lett. 94B, 495 (1980).

[7] P. Minkowski, Phys. Lett. B 67 (1977) 421; M. Gell-Mann, P. Ramond and R. Slansky, Pro- ceedings of the Supergravity Stony Brook Workshop, eds. P. van Niewenhuizen and D. Freed- man (New York, 1979); T. Yanagida, Proceedings of the Workshop on the Baryon Number of the Universe and Unified Theories, Tsukuba, Japan, 13-14 Feb 1979; R. N. Mohapatra and G. Senjanovic, Phys. Rev. Lett. 44, 912 (1980) .

[8] M. Magg and C. Wetterich, Phys. Lett. 94B, 61 (1980). G. Lazarides, Q. Shafi and C. Wetterich, Nucl. Phys. B 181, 287 (1981). R. N. Mohapatra and G. Senjanovic, Phys. Rev. D 23, 165 (1981). E. Ma and U. Sarkar, Phys. Rev. Lett. 80, 5716 (1998) hep-ph/9802445; W. Konetschny and W. Kummer, Phys. Lett. 70B, 433 (1977). J. Schechter and J. W. F. Valle, Phys. Rev. D 22, 2227 (1980). T. P. Cheng and L. F. Li, Phys. Rev. D 22, 2860 (1980).

[9] R. Foot, H. Lew, X. G. He and G. C. Joshi, Z. Phys. C 44, 441 (1989)

[10] S. Weinberg, Phys. Rev. Lett. 43, 1566, 1979

[11] S. Bhattacharya, B. Karmakar, N. Sahu and A. Sil, arXiv:1611.07419 [hep-ph].

[12] S. Bhattacharya, B. Karmakar, N. Sahu and A. Sil, Phys. Rev. D 93, no. 11, 115041 (2016) arXiv:1603.04776 [hep-ph]].

[13] S. Bhattacharya, S. Jana and S. Nandi, Phys. Rev. D 95, no. 5, 055003 (2017) arXiv:1609.03274 [hep-ph]].

[14] S. Bhattacharya, N. Sahoo and N. Sahu, Phys. Rev. D 93, no. 11, 115040 (2016) arXiv:1510.02760 [hep-ph]].

[15] S. Bhattacharya, S. Patra, N. Sahoo and N. Sahu, JCAP 1606, no. 06, 010 (2016) arXiv:1601.01569 [hep-ph]].

[16] N. Arkani-Hamed, S. Dimopoulos and S. Kachru, hep-th/0501082.

[17] R. Mahbubani and L. Senatore, Phys. Rev. D 73, 043510 (2006) hep-ph/0510064].

[18] F. D'Eramo, Phys. Rev. D 76, 083522 (2007) arXiv:0705.4493 [hep-ph]].

[19] R. Enberg, P. J. Fox, L. J. Hall, A. Y. Papaioannou and M. Papucci, JHEP 0711, 014 (2007) arXiv:0706.0918 [hep-ph]].

[20] G. Cynolter and E. Lendvai, Eur. Phys. J. C 58, 463 (2008) arXiv:0804.4080 [hep-ph]].

[21] T. Cohen, J. Kearney, A. Pierce and D. Tucker-Smith, Phys. Rev. D 85, 075003 (2012) arXiv:1109.2604 [hep-ph]].

[22] C. Cheung and D. Sanford, JCAP 1402, 011 (2014) arXiv:1311.5896 [hep-ph]].

[23] D. Restrepo, A. Rivera, M. Snchez-Pelez, O. Zapata and W. Tangarife, Phys. Rev. D 92, no. 1, 013005 (2015) arXiv:1504.07892 [hep-ph]].

[24] L. Calibbi, A. Mariotti and P. Tziveloglou, arXiv:1505.03867 [hep-ph].

[25] G. Cynolter, J. Kovacs and E. Lendvai, arXiv:1509.05323 [hep-ph].

[26] E. Aprile et al. XENON100 Collaboration, "Dark Matter Results from 225 Live Days of XENON100 Data," Phys. Rev. Lett. 109, 181301 (2012) arXiv:1207.5988 [astro-ph.CO]].

[27] D. S. Akerib et al., arXiv:1608.07648 [astro-ph.CO].

[28] E. Aprile et al. [XENON Collaboration], JCAP 1604, no. 04, 027 (2016) arXiv:1512.07501 [physics.ins-det]].

[29] C. Patrignani et al. (Particle Data Group), Chin. Phys. C40, 100001 (2016).

[30] K. Griest and D. Seckel, Three exceptions in the calculation of relic abundances, Phys. Rev. D 43, 3191 (1991);

[31] G. Belanger, F. Boudjema, A. Pukhov and A. Semenov, Comput. Phys. Commun. 180, 747 (2009) arXiv:0803.2360 [hep-ph]].

[32] D. Tucker-Smith and N. Weiner, Phys. Rev. D 64, 043502 (2001) hep-ph/0101138.

[33] Y. Cui, D. E. Morrissey, D. Poland and L. Randall, JHEP 0905, 076 (2009) arXiv:0901.0557 [hep-ph]].

[34] C. Arina and N. Sahu, Nucl. Phys. B 854, 666 (2012) arXiv:1108.3967 [hep-ph]].

[35] C. Arina, J. O. Gong and N. Sahu, Nucl. Phys. B 865, 430 (2012) arXiv:1206.0009 [hep-ph]].

[36] C. Arina, R. N. Mohapatra and N. Sahu, Phys. Lett. B 720, 130 (2013) arXiv:1211.0435 [hep-ph]].

[37] M. W. Goodman and E. Witten, Phys. Rev. D 31, 3059 (1985).

[38] R. Essig, Phys. Rev. D 78, 015004 (2008) arXiv:0710.1668 [hep-ph]]. 